Research Article

\title{
Quality Evaluation Method of a Mathematics Teaching Model Reform Based on an Improved Genetic Algorithm
}

\author{
Yun Yang \\ School of Physical and Mathematical Sciences, Nanjing Tech University, Nanjing, Jiangsu 211800, China \\ Correspondence should be addressed to Yun Yang; yangyun@nuaa.edu.cn
}

Received 5 July 2021; Revised 26 July 2021; Accepted 10 August 2021; Published 19 August 2021

Academic Editor: Mian Ahmad Jan

Copyright (c) 2021 Yun Yang. This is an open access article distributed under the Creative Commons Attribution License, which permits unrestricted use, distribution, and reproduction in any medium, provided the original work is properly cited.

\begin{abstract}
The poor comprehensiveness of the evaluation indexes of quality evaluation methods for the traditional college mathematics teaching model reform results in low accuracy of the evaluation outcomes. In this paper, aiming at this problem, a quality evaluation method for the college mathematics teaching model reform, based on the genetic algorithm, is proposed. The simulated annealing algorithm uses the weighted comprehensive objective evaluation multiobjective optimization effect that can effectively improve the accuracy of the evaluation results. In the training process, the gradient descent back-propagation training method is used to obtain new weights for the quality evaluation of college mathematics teaching mode reforms and to score various indicators and evaluate the indicators. The mean value of the outcomes is the result of mathematics teaching quality evaluation. The experimental results show that the training error of the convolutional network of the proposed method is significantly small. Based on the genetic algorithm that improves the convolutional network training process, the obtained quality evaluation outcomes are higher in accuracy, better in goodness of fitness function, and considerably lower than other state-of-the-art methods. We observed that the improved genetic algorithm has a more than $90 \%$ goodness of fit and the error is significantly lower, that is, 0.01 to 0.04 , than the classical genetic algorithm.
\end{abstract}

\section{Introduction}

With the continuous adjustment of college talent training goals, college mathematics education and teaching have changed from the early learning of basic theoretical knowledge system to the ability of mathematics innovation and application. Under these circumstances, the content and form of mathematics education and teaching in colleges and universities need to be optimized to meet the requirements of innovation and practice in mathematics education and teaching in the new era. Affected by the traditional teaching concepts and other factors, the content and form of mathematics education and teaching in China's colleges and universities have not been innovated with the development of society, which has caused college students to lack interest in mathematics classroom teaching. Therefore, students' enthusiasm and initiative to participate in classroom teaching are relatively high to low. Traditional mathematics teaching is mainly based on the basic theoretical knowledge, but this ignores the cultivation of college students' practical application ability in mathematics. For these reasons, college students cannot adapt to the actual needs of social development, which affects the overall development of college students and provides less employment opportunities for college students [1]. In response to this problem, college mathematics education and teaching should take the needs of mathematics professionals in the new era of social development as the starting point, enrich the teaching content, innovate mathematics education and teaching methods, strengthen the training of college students' mathematics practice and application ability, and promote college students' comprehensive mathematics literacy.

High-quality teaching is not only the fundamental purpose of the college or university but also the basic task of all educational institutions including colleges and universities, and it is also the foundation of all educational institutes. Colleges and universities must strictly establish the long-term concept of "survive by quality and develop by 
quality," supervise and control the quality of teaching, focus on teaching observation and evaluation, and continue to provide talents for the society [2]. Nowadays, with the continuous development and updated technologies in colleges and universities, the teaching reform can become more dynamic, and the effective use of Internet along with the education technology can also expand teachers and students to a certain extent [3]. Few researchers have already investigated this issue, but they still failed to provide an effective solution and reform model.

Literature [4] aimed at the evaluation of classroom teaching quality in colleges and universities and proposed an auxiliary teaching quality evaluation model based on active learning support vector machines. Comprehensive consideration of various actual conditions is used in constructing an evaluation index system for classroom teaching quality. Adopting active learning support vector machine establishes a classroom teaching quality evaluation model. Literature [5] designs a university teaching quality evaluation model based on data mining algorithms. This model first studies and analyzes the current literature on teaching quality evaluation in universities and establishes the impact of university teaching quality evaluation factors. Next, the model collects data based on those factors that may affect the teaching quality of colleges and universities. The authors then use experts to determine the teaching quality level of colleges and universities and establish learning samples for college teaching quality evaluation. Finally, the BP neural network of the data mining technology is introduced to train the learning samples to form the teaching quality of colleges' and universities' evaluation model.

In the process of evolution, the feasible solution set of genetic algorithm retains the better solution with the corresponding objective function value with a certain probability through the selection operation. Moreover, this eliminates the corresponding solution with the worse objective function value and constructs it through the crossover operation and the mutation operation. In this way, the entire feasible solution set can gradually get closer and closer to the global optimal solution through continuous iteration and evolution [6]. When the whole algorithm is over, decode the most adaptable individuals in the last generation of the population for an optimized feasible solution. Internet technology has spread to all corners of people's lives that not only brings convenience to people but also has a huge impact on traditional mathematics teaching models. The huge impact has also brought other advanced methods and platforms to the reform and time of meta solution that can be obtained.

In this paper, we propose a method for evaluating the quality of college mathematics teaching model reform based on an improved genetic algorithm. The basic process of genetic algorithm is described. On this basis, an improved simulated annealing algorithm is obtained by simulating the annealing process in physical thermodynamics. Furthermore, the main points of the reform of mathematics teaching mode in colleges and universities are analyzed, and the quality evaluation system of mathematics teaching mode reform is constructed. Then, we train the convolutional neural network through the simulated annealing algorithm and use the convolutional neural network optimized, through the simulated annealing algorithm, for evaluation and learning. Finally, we apply it to the evaluation of mathematics teaching quality in colleges and universities. The convolutional neural network training divides the quality of mathematics teaching into different indicators to carry out evaluation. The inputs of these indicators are then fed into each convolutional network, which scores each indicator. We then use the average of the evaluation outcomes obtained through these indicators as the result of the entire mathematics teaching quality evaluation model. The following are the main innovations points of the research presented in this manuscript:

(1) Improving the traditional genetic algorithm by simulating the annealing process in physical thermodynamics

(2) Analyzing the reform content of mathematics teaching mode in colleges and universities

(3) Formulating the quality evaluation system of mathematics teaching mode reform according to the reform content

(4) Using genetic algorithm to improve the convolutional neural network.

The structure of the remaining part of this paper is as follows: Section 2 describes the details of the related work in the context of genetic and simulated annealing algorithms. In Section 3, we discuss the evaluation of the reform quality of mathematics teaching mode in colleges and universities based on an improved genetic algorithm. A new variant of the genetic algorithm is suggested. Section 4 is about experimental setup and results. Finally, Section 5 concludes this paper along with directions for further research and investigation.

\section{Improved Genetic Algorithm}

2.1. The Basic Process of the Classical Genetic Algorithm. The standard genetic algorithm generally includes three basic operations, that is, (1) a selection operation, (2) crossover operation, and (3) mutation operation [7]. These operations are briefly described in subsequent paragraphs.

(1) The main purpose of the selection operation is to select individuals with good performance and eliminate individuals with the poor performance. At the same time, to keep the size of the population unchanged, it consists of two steps: (i) first, calculate the fitness value of everyone in the population and (ii) then select individuals in the population based on the fitness value according to a certain method.

(2) The selection operation is just to copy the individuals with good performance so that they can occupy a larger proportion in the population and eliminate the individuals with poor performance from the population, and this process does not create any new individuals. The crossover operation is to generate a 
new individual by exchanging part of the genetic information possessed by the two parent individuals according to certain rules. To keep some well-behaved individuals in the population intact, not every individual will participate in the crossover operation [8]. In the actual operation, first, generate a random number within the range of 0 to 1 and then judge whether the value of this random number is greater than the crossover's preset probability. In case the value is smaller than the threshold values, then the selected individual will participate in the crossover operation, otherwise not.

(3) The performance of the offspring individuals generated by the crossover operation may not be better than that of the parent individuals. This is due to the fact that the effective gene is missing, and the algorithm falls into a local optimal solution. To overcome this situation, it is necessary to perform mutation operations on some individuals in the population, that is, to change their own structure to a certain degree according to certain rules, so that the offspring individuals can show the traits that the parent individuals do not have. The standard genetic algorithm mainly has the following steps, as illustrated in Algorithm 1. The flowchart of the standard genetic algorithm is shown in Figure 1.

\subsection{Principle and Basic Process of the Simulated Annealing} Algorithm. The simulated annealing algorithm is a general probabilistic heuristic algorithm, which is a simulation of the annealing process in physical thermodynamics and is used to search for the global optimal solution of the optimization problem in a huge solution space. The standard simulated annealing algorithm mainly has the steps as illustrated in Algorithm 2. The flowchart of the standard simulated annealing algorithm is shown in Figure 2.

It can be seen from the process in Figure 2 that the following factors affect the outcomes of the algorithm. The simulated annealing algorithm has the following major advantages over the classical genetic algorithm:

(1) The initial value is very robust, which reduces the quality requirements for the initial solution

(2) With progressive convergence, the algorithm can finally converge to the global optimal solution with a $100 \%$ probability

(3) It is potentially very easy to implement and has a wide range of applications in other fields

\section{Evaluation of the Reform Quality of Mathematics Teaching Mode in Colleges and Universities Based on an Improved Genetic Algorithm}

In this section, we briefly describe the methods of optimizing mathematics teaching and optimize them using an improved variant of the classical genetic algorithm.
3.1. Analysis of the Reform of Mathematics Teaching Mode in Colleges. For higher mathematics, the reform of mathematics teaching mode can play an important role in cultivating students' logical thinking and practical abilities. For the current education, if the teacher only knows to blindly pay attention to the theoretical aspects of teaching, it will make the students' understanding and thinking ability in the classroom very rigid, and therefore, they will only blindly follow the teacher's footsteps to learn the basics. Therefore, in such circumstances, the innovative thinking abilities of the students cannot be cultivated. The reform of mathematics teaching mode in educational institutes including colleges and universities mainly includes the contents that are discussed in subsequent subsections.

\subsubsection{Methods of Optimizing Mathematics Teaching and} Training Concepts. The injecting teaching method is a widely used and well-known methodology in mathematics teaching of colleges and universities. Under the background of the current new era, traditional teaching methods should be effectively changed and updated [9]. On the one hand, colleges and universities should abandon the original theoretical and test-oriented teaching models and cultivate students' innovative ability and application ability to adapt to social development as the current teaching goal. On the other hand, to make the teaching content practical, a diversified teaching system should be continuously constructed and some practical teaching methods and evaluation methods need to be infiltrated into the teaching content to ensure quality to a certain extent. The teaching content can be linked to the actual life and then lay a solid foundation for the future development of the students.

3.1.2. Effectively Optimize the Teaching System. In this regard, it is essential to understand the characteristics of the instrumental subjects of higher mathematics, to reform the syllabus and teaching content. Furthermore, it is also needed to make the students' majors and characteristics that can coordinate and cooperate with each other. In this way, the importance of mathematics could possibly be strengthened. In addition, in the construction of the teaching system, it is necessary to ensure the subjectivity of students in teaching, and it is also necessary to effectively stimulate the enthusiasm of students in the process of teaching and then enhance the initiative of students. In the teaching of advanced mathematics, teachers should vigorously encourage students to think, and fully understand the class within the classroom activities, to choose different teaching methods for different students, and effectively improve the quality of teaching.

\subsubsection{Effectively Reform the Current Teaching Evaluation and} Assessment Methods. At present, the main method of evaluating mathematics teaching in domestic colleges and universities is the evaluation of students' performance. Under the new situation, the method of teaching reform must break through the traditional one-size-fits-all teaching evaluation method [10]. For example, in the assessment of 
Step 1: randomly generate several initial feasible solutions according to a certain method and use the set of them as the initial population.

Step 2: evaluate everyone in the population, calculate their fitness value, and then determine whether it meets the optimization criteria of the algorithm. If it does, the current best-performing individual is the optimal solution and output solve the results and end the algorithm; otherwise, go to Step 3.

Step 3: according to the fitness of the individuals in the population, use a certain method to select the survival of the fittest in the population. The greater the fitness of the individual, the more likely it is to be selected; otherwise, the more likely it is to be eliminated. Step 4: use a certain method to perform crossover operations on the population with the set crossover probability.

Step 5: use a certain method to perform mutation operations on the population with the set mutation probability.

Step 6: go to Step 2.

Algorithm 1: The standard genetic algorithm.

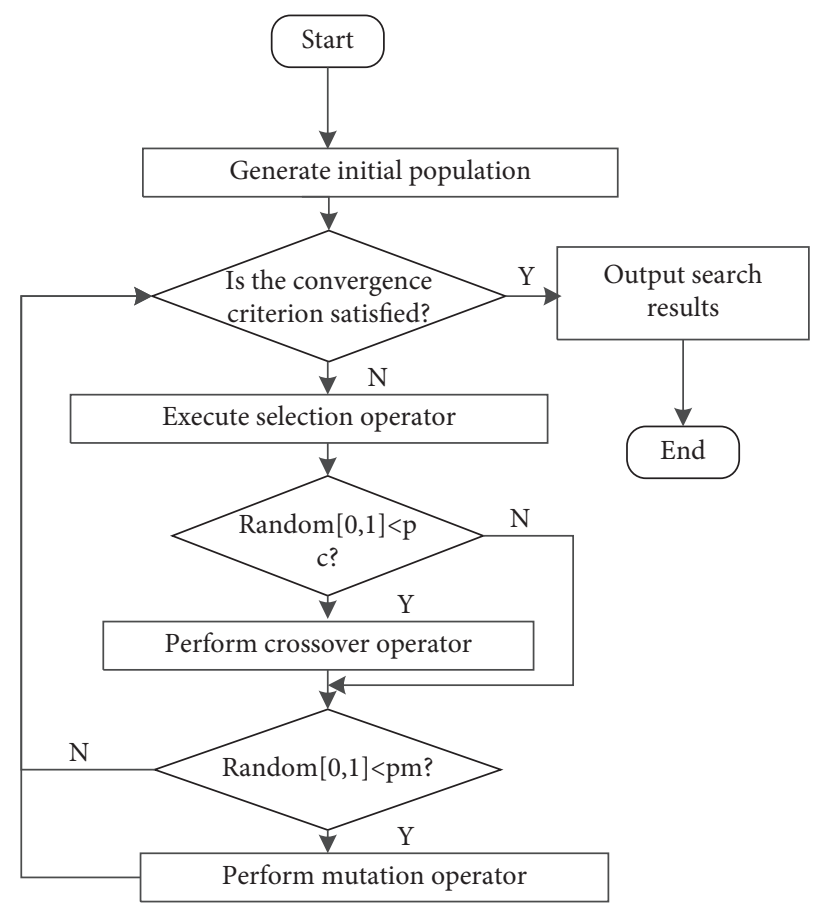

Figure 1: The flowchart of the standard genetic algorithm process [7].

Step 1: set the initial temperature $t_{0}$, generate an initial feasible solution $x_{i}$ according to a certain method, and calculate its corresponding energy value $E\left(x_{i}\right)$.

Step 2: determine whether the termination condition of the outer loop is satisfied. If it is satisfied, the current optimal solution is the required optimal solution. Hence, output the result and end the algorithm; otherwise, go to Step 3.

Step 3: determine whether the termination condition of the inner loop is met; if it is met, then update the temperature of the system and go to Step 2; otherwise, go to Step 4.

Step 4: starting from the current solution $x_{i}$, generate a new feasible solution $x_{j}$ according to a certain method and calculate its corresponding energy value $E\left(x_{j}\right)$.

Step 5: if $E\left(x_{i}\right)>E\left(x_{j}\right)$, then accept $x_{j}$ as the current optimal solution; otherwise, accept $x_{j}$ as the current optimal solution with probability $\exp \left[E\left(x_{i}\right)-E\left(x_{j}\right) / k t\right]$.

Step 6: go to Step 3.

Algorithm 2: The standard simulated annealing algorithm.

students, it is necessary to add some practical skills exercises, which will make the assessment space wider, so that students can apply the knowledge content they have learned through practice and think to answer. In terms of assessment, there are certain differences among various students in the classroom, so teachers need to adopt different methods for 


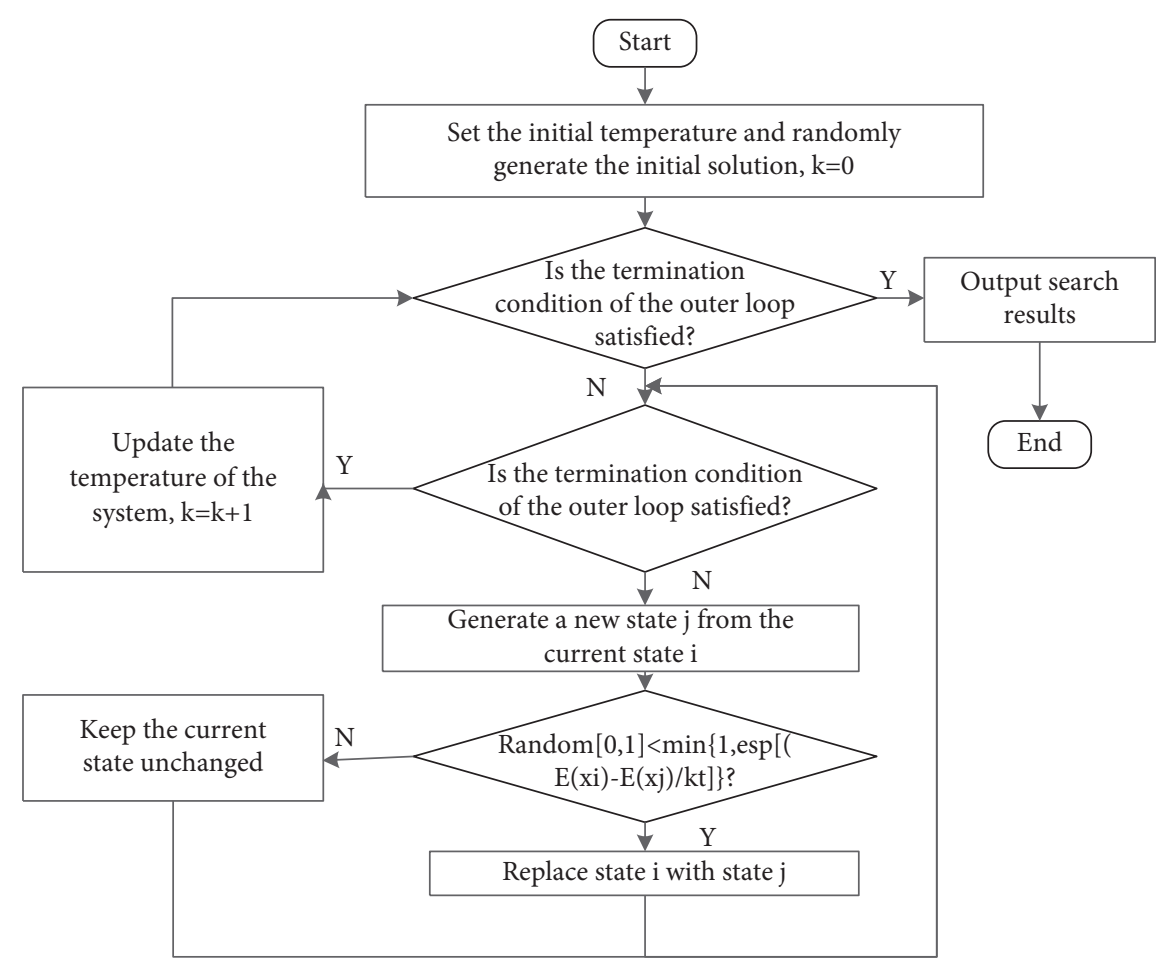

Figure 2: The flowchart of the standard simulated annealing algorithm flow $[7,8]$.

different students as appropriate to ensure fair assessment of the course catalog and teaching quality.

\subsection{The Quality Evaluation System of the Reform of Mathe-} matics Teaching Mode. The quality evaluation index system of mathematics teaching model reform is an important determinant of whether the quality evaluation of mathematics teaching model reform in colleges and universities is scientific, fair, and reasonable. The evaluation of the quality of mathematics teaching model reform in higher education schools is a very complex issue. The different types of courses and student types make it difficult to find a universally applicable mathematics teaching model reform quality evaluation system that can be used to evaluate the quality of teachers' mathematics teaching model reforms in all colleges and universities. For different types of higher education schools, according to their own development and future goals, they can adopt more targeted and more suitable evaluation indicators to make the mathematics teaching model reform quality evaluation system more accurate and scientific, and to improve the quality of the mathematics teaching model reform. The evaluation work plays a very important role. The rationality of the comprehensive evaluation results of the quality of mathematics teaching model reform can enable school leaders, administrations, and the staff of the mathematics teaching model reform quality evaluation management department. Moreover, this will also help all the teachers themselves to timely understand and discover the existence of their own mathematics teaching model reforms and make appropriate adjustments within time [11]. It has an irreplaceable effect on the development of colleges and universities, the cultivation of students, and the continuous improvement of teachers' mathematics teaching model reform.

The establishment of a quality evaluation system for mathematics teaching model reform should be a mathematics teaching model reform model and method that can promote individual development, respect commonality, pay attention to individuality, reflect the common law of mathematics teaching model reform, reflect the mathematics of different types and levels of college teachers, and the characteristics of teaching mode reform. Currently, the establishment of a diversified, reasonable, and distinctive mathematics teaching model to reform the quality evaluation standard system has a key role and significance [12]. It can eliminate the idleness and waste of educational resources in time and realize the effective allocation of resources based on the research results. The current mathematics teaching model reform quality evaluation system is based on the problems in the establishment process and follows the principles of comprehensiveness, directionality, motivation and improvement, objectivity, and subjectivity. For different types of courses, such as basic theory courses, the mathematics teaching model reforms practical courses, comprehensive courses, etc., according to the unified standards of the school. According to the characteristics of courses, subjects, grades, etc., using different evaluation indicators, or using different weights for evaluation, using artificial intelligence, etc. Note that the nonlinear problems are more objective evaluation methods and could improve the problems of the single evaluation type of teacher mathematics teaching model reform quality, the unreasonable proportion of evaluation indicators, and the strong 
subjective factors of evaluation methods [13]. Based on the above analysis, we construct the quality evaluation index system of mathematics teaching mode reform, as shown in Table 1.

As the output of the neural network, the quality evaluation outcomes of the mathematics teaching model reform are divided into five different grades: (a) excellent, (b) good, (c) intermediate, (d) pass, and (e) fail. The score ranges of each grade of the teaching quality evaluation outcomes, in this article, are shown in Table 2.

\subsection{Construction of a Quality Evaluation Model for the Reform} of Mathematics Teaching Mode Based on an Improved Genetic Algorithm. To sum up, the improved genetic algorithm, that is, simulated annealing algorithm, is used to evaluate the reform quality of mathematics teaching mode in colleges and universities. The steps of the simulated annealing algorithm are illustrated in Algorithm 3.

The simulated annealing algorithm uses weighted comprehensive targets to evaluate the effect of multiobjective optimization. Based on the randomness of the weights, through repeated or parallel operation of the algorithm, the optimal solution of the pareto boundary in each direction is obtained, and multiple selection targets are further assigned to the decision [14].

At this stage, the convolutional neural network has achieved certain results in the evaluation of teaching quality, which can effectively improve the accuracy of the evaluation results. In the evaluation of mathematics teaching quality, the convolutional neural network is trained through the simulated annealing algorithm, and the modified linear unit is used to obtain its operating function [15], namely:

$$
C_{j+1}=\max \left(0, W_{j} \otimes C_{j}+B_{j}\right) .
$$

Among them, $C_{j}$ describes the evaluation index value obtained in a particular convolutional network layer, i.e., $j$; $W_{j}$ describes the weight value obtained when the convolutional layer is $j ; B_{j}$ describes the bias parameter of the convolutional neural network for layer $j ; \otimes$ represents the convolution operation, which indicates the maximum extreme value of the running function, and the pooling method selects the maximum extreme value pooling to obtain

$$
C_{j+1}=\max _{R} C_{j}
$$

Among them, $R$ represents any pooling range in the evaluation index, while $C_{j+1}$ represents the largest extreme value within the range.

The selected index data is fed into the convolutional neural network for network training purpose. Use the norm method, as given by equation (3), to set the loss function in the network training process.

$$
J=\sqrt{\|f(x, W)\|^{2}} .
$$

Among them, $x$ represents the input training evaluation index; $W$ represents the network weight; and $f(x, W)$ represents the input index $x$ the convolutional network sends the evaluation score value. After minimizing $J$ to train all convolutional nerves, the process uses the gradient descent back-propagation training method to obtain brandnew weights, as given by

$$
\begin{aligned}
& \Delta_{i+1}=m \times \Delta_{i}-\eta \times \frac{\partial L}{W_{i}^{j}}, \\
& W_{i+1}^{j}=W_{i}^{j}+\Delta_{i+1}-\lambda \times \eta \times W_{i}^{j} .
\end{aligned}
$$

Among them, $m$ represents the momentum factor in the gradient descent backpropagation; $\eta$ represents the learning probability; $i$ represents the number of network training; $\Delta_{i+1}$ represents the gradient increment with $i$ training times; and $\lambda$ represents the weight attenuation value.

In the training process, we set the learning probability within the range of $[0.001,0.001]$. After training, we take the weight of the last iteration as the weight value of the following and next mathematics teaching quality index evaluation.

Convolutional neural network training divides the quality of mathematics teaching into different indicators for evaluation and inputs these indicators into each convolutional network. Then the network scores each indicator, which is recorded as $q_{i}$, to obtain different indicator scores. Finally, we use the mean value of the index evaluation outcomes as the result of the whole mathematics teaching quality evaluation, as illustrated by the following equation:

$$
Q=\frac{1}{N} \sum_{i}^{N} q_{i}
$$

Among them, $Q$ represents the result of the mathematics teaching quality evaluation method, while all other notations are as described earlier.

\section{Experimental Results and Analysis}

4.1. Experimental Settings. To verify the effectiveness of the quality evaluation method of the college mathematics teaching model reform designed based on the improved version of the classical genetic algorithm, an online teaching course was selected as the test object. A total of 5,000 people attended the online teaching course. Students gave their feedbacks on the evaluation texts after completing the class. We refined the collected data through deleting useless evaluation texts and junk evaluation texts and considered a total of 4,500 valid evaluation texts. The experiment selects the first 4,000 sets of data among the 4,500 sets of data as training samples, while the remaining 500 sets were used as experimental test data. Furthermore, the corresponding evaluation target is used as the expected output value. The teaching quality evaluation error was set to 0.0001 . We used the MATLAB_7.0 tool to write the program and implement the proposed algorithms. Similarly, we also carried out genetic algorithm to improve the training process of the convolutional neural network. Three evaluation metrics are used to compare the outcomes of various methods, i.e., (1) training time; (2) mean square error; and (3) goodness of 
TABLE 1: The quality evaluation index system of mathematics teaching model reform.

\begin{tabular}{|c|c|c|}
\hline First-level evaluation index & Secondary evaluation index & Index code \\
\hline \multirow{3}{*}{ Teacher quality } & Clear educational goals & $\mathrm{X} 1$ \\
\hline & Solid professional knowledge & $\mathrm{X} 2$ \\
\hline & The level of teaching explanation & $\mathrm{X} 3$ \\
\hline \multirow{3}{*}{ Teaching attitude } & Counseling and answering questions patiently and positively & $\mathrm{X} 4$ \\
\hline & The lectures are conscientious and contagious & $\mathrm{X} 5$ \\
\hline & Rigorous attitude and strive for perfection & $\mathrm{X} 6$ \\
\hline \multirow{4}{*}{ Teaching content } & Conceptual theory is accurate & $\mathrm{X} 7$ \\
\hline & Full of content and focus on ability & $\mathrm{X} 8$ \\
\hline & Connect with reality and focus on practice & X9 \\
\hline & Depth and breadth of expertise & $\mathrm{X} 10$ \\
\hline \multirow{4}{*}{ Teaching method } & Good at inspiring and guiding thoughts & $\mathrm{X} 11$ \\
\hline & Various methods and appropriate use & $\mathrm{X} 12$ \\
\hline & Pay attention to individuality and teach students in accordance with their aptitude & $\mathrm{X} 13$ \\
\hline & Focus on stimulating innovation & $\mathrm{X} 14$ \\
\hline \multirow{4}{*}{ Teaching effect } & Self-study ability and interest in learning & $\mathrm{X} 15$ \\
\hline & Basic knowledge understanding & $\mathrm{X} 16$ \\
\hline & Analyze and solve problems & $\mathrm{X} 17$ \\
\hline & Comprehensive quality and innovation ability & $\mathrm{X} 18$ \\
\hline
\end{tabular}

TABLE 2: Evaluation result grade.

\begin{tabular}{lc}
\hline Grade & Score range \\
\hline Excellent & {$[90,100]$} \\
Good & {$[80,89]$} \\
Medium & {$[70,79]$} \\
Timely & {$[60,69]$} \\
Failed & {$[0,59]$} \\
\hline
\end{tabular}

fitness function [16]. Note that these evaluation metrics are most frequently used in the context of evolutionary optimization methods [17]. The experiments are performed on a Core i-7 CPU machine having 16 GB of memory and a GPU unit.

\subsection{Improved Training Results of Convolutional Neural} Networks Based on Genetic Algorithms. After the construction of the convolutional neural network structure is completed, we trained the network so that it can evaluate the quality index of mathematics teaching in colleges and universities. The training samples and the evaluation indicators corresponding to the samples need to be prepared in advance. According to the obtained indicators, this network model training is carried out using the experimental parameters as described earlier. The training results of the convolutional neural network, in terms of mean square error and number of training times, are shown in Figure 3.

According to Figure 3, the method suggested in this paper (the improve genetic algorithm) regards the modified linear unit as the running function in the entire neuron. Therefore, after 10 trainings periods, the accuracy requirement of 0.01 can be achieved. Similarly, the accuracy requirement of 0.001 can be completed after 20 training periods and the accuracy of 0.0001 can be completed after 50 training periods, respectively.
4.3. Comparison of the Evaluation Outcomes. The method suggested in this paper was compared with the methods proposed in the literature $[4,5]$. The experimental parameters were kept the same during the comparison of the quality evaluation training outcomes of college mathematics teaching mode reform, and the results are shown in Table 3. Two evaluation metrics, i.e., the training time and goodness of fitness function, were used to evaluate the performance of various approaches and algorithms. The training times denote how many times the experiments were run. We considered variations in this metric with a repeated increase of 50. Overall, these values ranged from 50 to 500 to denote 10 iterations. Note that, in Table 3, error means mean square error. All other parameters along with machine specification were kept the same to ensure the accuracy of the outcomes.

From the evaluation and comparison of the 500-sample data in Table 3, the maximum error of the evaluation result of the method in this paper is 0.04 , which is less than the evaluation error of the literature methods, because the literature methods do not use the gradient descent backpropagation method and cannot update the weights in real time. The training process is still using the previous weights. From our experimentation, this can be concluded that there is a large error in the teaching quality evaluation method. Therefore, the evaluation results obtained by the method suggested in this paper are very close to the real evaluation outcomes.

To further verify the effectiveness of the quality evaluation method of the reform of college mathematics teaching model based on the improvement of classical genetic algorithm, the analysis is carried out from the angle of goodness-of-fit function. The larger the result of the goodness of fit is, the closer the evaluation index result is to the real result. The experiment still chooses the literature methods as the control group of the proposed method. The change of the goodness of fitness function curve is shown in Figure 4. 
Step 1: let the initial feasible solution and weight be randomly generated, then the initial feasible solution is $X_{1}, \ldots, X_{L}$; there are $L$ in total, the weight is $w_{1}, \ldots, w_{K}$, and the number is $K$, where the value range of $w_{i}$ is $[0,1]$ and satisfies $\sum_{i=1}^{K} w_{i}=1$. Through the evaluation of the single optimization goal $f_{i}$ and the comprehensive goal $F=\sum_{i=1}^{K} w_{i} f_{i}$, the initial temperature $t_{0}$ is clarified. Based on the comprehensive goal $F=\sum_{i=1}^{K} w_{i} f_{i}$, it is clear that the optimal solution $X *$ is reached, and after it is regarded as the current solution $X$, it meets $k=0$.

Step 2: based on the neighborhood function, generate a new solution $X^{\prime}$ according to the current solution $X$ and terminate when the solution is feasible to complete the evaluation of each optimization objective and comprehensive objective.

Step 3: if there is $F_{X^{\prime}}<F_{X}$, then replace the current solution $X$ with the new solution $X^{\prime}$ and then the current solution at this time becomes $X^{\prime}$, and if $F_{X^{\prime}}<F_{X *}$, then replace the optimal solution $X *$ with the new solution $X^{\prime}$; on the contrary, if there is $\exp \left[F_{X}-F_{X^{\prime}} / t_{k}\right]>\operatorname{random}(0,1)$, then replace the current solution $X$ with the new solution $X^{\prime}$. If not, keep the current solution $X$. Step 4: the current temperature sampling criterion meets the state implementation judgment. If it meets the sampling criterion, proceed to the next process; otherwise, return to the second step.

Step 5: implement temperature reduction treatment. There are $t_{k+1}=\lambda t_{k}$ and $k=k+1$.

Step 6: if the algorithm termination condition is reached, the search stops and $X *$ is output; otherwise, it returns to the second step.

Algorithm 3: The improved genetic algorithm.

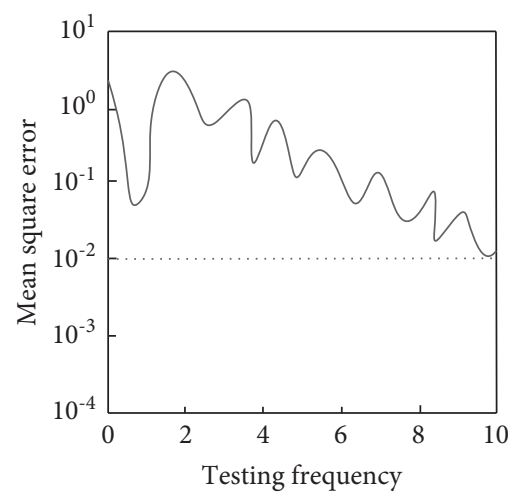

(a)

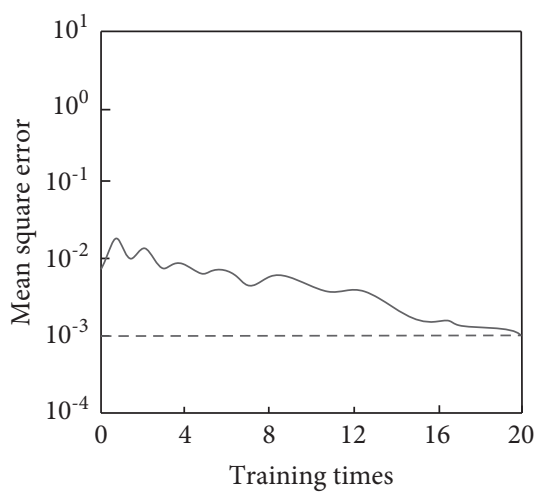

(b)

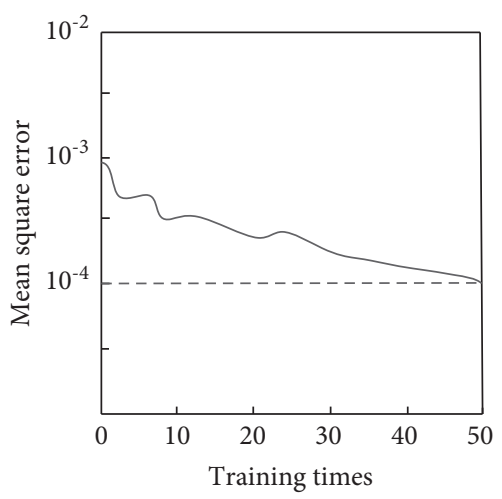

(c)

FIGURE 3: Convolutional neural network training error (error situation when the evaluation error is 0.01 (a), 0.001 (b), and 0.0001 (c)).

TABLE 3: Comparison of teaching quality evaluation results.

\begin{tabular}{lccccccc}
\hline \multirow{2}{*}{ Training times } & \multicolumn{2}{c}{ Method of this article } & \multicolumn{2}{c}{ Literature [4] method } & \multicolumn{2}{c}{ Literature [5] method } \\
& The assessed value & Error & The assessed value & Error & The assessed value & Error & Actual value \\
\hline 50 & 7.49 & 0.01 & 7.58 & 0.08 & 7.43 & 0.07 & 7.5 \\
100 & 6.87 & 0.03 & 6.95 & 0.05 & 6.96 & 0.06 \\
150 & 7.12 & 0.02 & 7.05 & 0.05 & 7.19 & 0.09 \\
200 & 7.32 & 0.02 & 7.37 & 0.07 & 7.24 & 0.06 \\
250 & 8.14 & 0.04 & 8.02 & 0.08 & 8.03 & 0.07 \\
300 & 8.49 & 0.01 & 8.44 & 0.06 & 8.59 & 0.09 \\
350 & 6.38 & 0.02 & 6.3 & 0.10 & 6.32 & 0.08 \\
400 & 5.86 & 0.04 & 5.82 & 0.08 & 5.81 & 8.1 & 0.09 \\
450 & 7.61 & 0.01 & 7.5 & 0.10 & 7.7 & 0.10 \\
500 & 9.32 & 0.02 & 9.39 & 0.09 & 9.35 & 7.9 \\
\hline
\end{tabular}

It can be clearly observed from Figure 4 that the goodnessof-fit curve obtained by the method proposed in this paper is higher than the curve of goodness of fit obtained by the literature methods. This is because the quality evaluation method of college mathematics teaching model reform based on genetic algorithm is almost subjective. Evaluation removal makes the evaluation result comprehensive; the genetic algorithm improves the convolutional neural network and uses the gradient descent back-propagation method, which can calculate the suitable gradient of the training sample in each iteration. This reduces the error of the actual teaching quality evaluation outcomes. Therefore, the results of the method suggested in this paper in terms of teaching quality evaluation are excellent. 


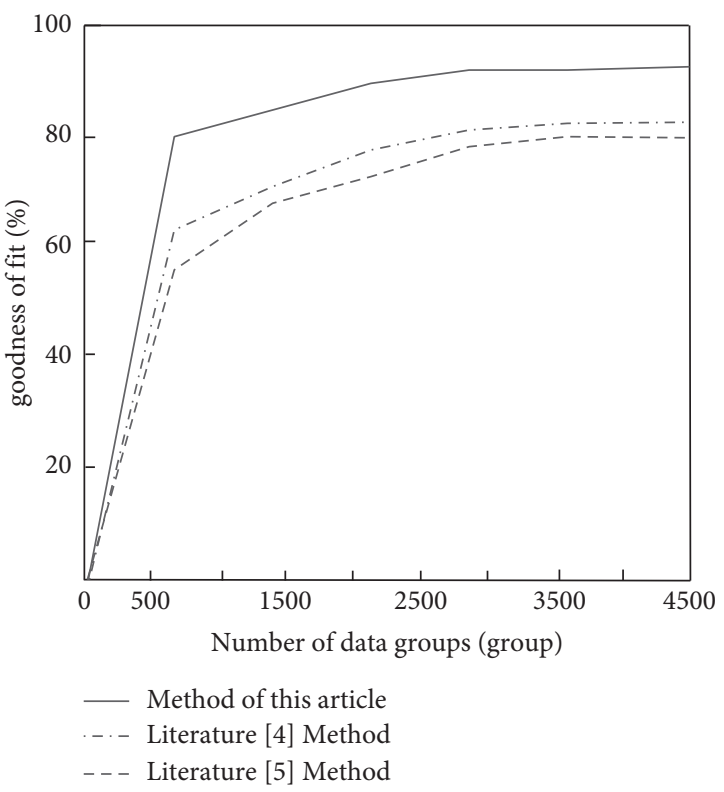

Figure 4: Comparison results of goodness of fit.

\section{Conclusions and Future Work}

With the continuous deepening and development of the college education reform and the increasing demand for applied talents in the society, at present, colleges and universities are facing huge difficulties and challenges in the training mechanism of mathematics talents. With the continuous development and progress of the times, how to effectively reform and innovate college mathematics is a difficult problem that needs to be solved effectively. Teaching quality evaluation is a very complex and fuzzy nonlinear process. In this process, multiple factors and variables are involved. Therefore, the establishment of mathematical models is more complicated, and traditional teaching quality evaluation methods are no longer fully competent to solve the issue. Only by applying the correct intelligent optimization algorithms to a reasonable teaching quality evaluation system can result in a scientific and reliable teaching quality evaluation model. In this paper, we proposed a method to evaluate the quality of college mathematics teaching mode reform based on an improved genetic algorithm.

Compared with traditional genetic algorithm, the simulated annealing algorithm has improved the diversity of new generations, and the performance of optimized neural network has been significantly improved. The improvement of mutation probability enhances the ability and speed of genetic algorithm to search for the global optimal solution, and the teaching quality evaluation model has more efficient convergence efficiency and more accurate prediction accuracy. The experimental results showed that the training error of the convolutional network of the proposed method is very small, the accuracy of the teaching quality evaluation outcomes is higher, and the goodness of fitness function value is better than other closest rivals. It can reduce the error of the actual teaching quality evaluation outcomes and can obtain a more accurate university mathematics teaching model reform quality assessment result. In the future, we will evaluate the model using other evolutionary algorithms including the well-known particle swarm optimization (PSO) and its various variants.

\section{Data Availability}

The data used to support the findings of this study are available from the corresponding author upon request.

\section{Conflicts of Interest}

The author declares that he has no conflicts of interest.

\section{References}

[1] L. Boulton, P. J. Olver, B. Pelloni, and D. A. Smith, "New revival phenomena for linear integro-differential equations," Studies in Applied Mathematics, vol. 31, no. 5, pp. 21-35, 2021.

[2] B. Deng, "Word order detection in english classroom teaching based on improved genetic algorithm of block coding," Journal of Intelligent and Fuzzy Systems, vol. 40, no. 6, pp. 1-12, 2020.

[3] D. Finkelhor, K. Walsh, L. Jones, K. Mitchell, and A. Collier, "Youth internet safety education: aligning programs with the evidence base," Trauma, Violence, \& Abuse, vol. 17, 2020.

[4] Y. Zhang, "Assisted teaching quality evaluation model based on active learning support vector machine," Modern Electronics Technique, vol. 42, no. 7, pp. 112-114, 2019.

[5] Y. Li, "Design of university teaching quality evaluation model based on data mining algorithm," Modern Electronics Technique, vol. 43, no. 17, pp. 127-130, 2020.

[6] R. Ponciroli, N. E. Stauff, J. Ramsey, F. Ganda, and R. B. Vilim, "An improved genetic algorithm approach to the unit commitment/economic dispatch problem," IEEE Transactions on Power Systems, vol. 35, no. 5, 2020.

[7] A. Kumar, S. Verma, and R. Das, "Eigenfunctions and genetic algorithm based improved strategies for performance analysis and geometric optimization of a two-zone solar pond," Solar Energy, vol. 211, pp. 949-961, 2020.

[8] P. Dziwinski and L. Bartczuk, "A new hybrid Particle Swarm optimization and genetic algorithm method controlled by fuzzy logic," IEEE Transactions on Fuzzy Systems, vol. 28, no. 6, 2019.

[9] X. Peng and J. Dai, "Research on the assessment of classroom teaching quality with q -rung orthopair fuzzy information based on multiparametric similarity measure and combinative distance-based assessment," International Journal of Intelligent Systems, vol. 34, no. 7, pp. 1588-1630, 2019.

[10] M. Hujala, A. Knutas, T. Hynninen, and H. Arminen, "Improving the quality of teaching by utilising written student feedback: a streamlined process," Computers \& Education, vol. 157, Article ID 103965, 2020.

[11] A. Rezaeipanah, S. S. Matoori, and G. Ahmadi, "A hybrid algorithm for the university course timetabling problem using the improved parallel genetic algorithm and local search," Applied Intelligence, vol. 51, no. 1, pp. 467-492, 2020.

[12] A. Abdelhadi and M. Nurunnabi, "Engineering student evaluation of teaching quality in Saudi arabia," International Journal of Engineering Education, vol. 35, no. 1A, pp. 262-272, 2019.

[13] K. Tehlan, S. Chakraverty, P. Chakraborty, and S. Khapra, “A genetic algorithm-based approach for making pairs and 
assigning exercises in a programming course," Computer Applications in Engineering Education, vol. 28, no. 6, pp. 1708-1721, 2020.

[14] C. Lu, B. He, and R. Zhang, "Evaluation of english interpretation teaching quality based on GA optimized RBF neural network," Journal of Intelligent and Fuzzy Systems, vol. 40, no. 2, pp. 3185-3192, 2021.

[15] X. Jiang, "Online English teaching course score analysis based on decision tree mining algorithm," Complexity, vol. 2021, Article ID 5577167, 10 pages, 2021.

[16] I. Rahman, Z. Wang, W. Liu, B. Ye, X. Liu, and Z. Muhammad, "An n-state markovian jumping particle swarm optimization algorithm," Transactions on Systems, Man, Cybernetics: Systems, vol. 13, 2020.

[17] I. Rahman, M. Zakarya, M. Raza, and K. Rahim, "An n-state switching PSO algorithm for scalable optimization," Soft Computing, vol. 24, 2020. 